Research Paper

\title{
Effect of Chronic Administration of Forskolin on Glycemia and Oxidative Stress in Rats with and without Experimental Diabetes
}

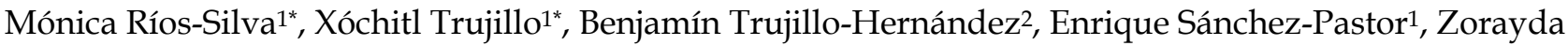 \\ Urzúa $^{3}$, Evelyn Mancilla ${ }^{1}$, and Miguel Huerta ${ }^{1}$ \\ 1. Enrico Stefani building, Centro Universitario de Investigaciones Biomédicas de la Universidad de Colima, Av. 25 de Julio \#965, colonia \\ Villas de San Sebastián, C.P. 28040. Colima, Colima, México. \\ 2. Unidad de Epidemiología Clínica, Instituto Mexicano del Seguro Social, Colima, Colima, México. \\ 3. Unidad de Medicina Familiar No. 19, Coordinación de Educación en Salud e Investigación Instituto Mexicano del Seguro Social, Colima, \\ Colima, México. \\ * Equal contribution.
}

\begin{abstract}
$\triangle$ Corresponding author: Dr. Miguel Huerta, Universidad de Colima, Centro Universitario de Investigaciones Biomédicas, Av. 25 de julio No. 965, Col. Villa San Sebastián, 28040-Colima, Colima. Phone. (312) 3161129 Fax: (312) 3161129 ext. 47452 e-mail: huertam@ucol.mx, huerta@ucol.mx.

() Ivyspring International Publisher. This is an open-access article distributed under the terms of the Creative Commons License (http://creativecommons.org/ licenses/by-nc-nd/3.0/). Reproduction is permitted for personal, noncommercial use, provided that the article is in whole, unmodified, and properly cited.
\end{abstract}

Received: 2013.II.0I; Accepted: 2014.02.27; Published: 2014.03.II

\begin{abstract}
Forskolin is a diterpene derived from the plant Coleus forskohlii. Forskolin activates adenylate cyclase, which increases intracellular CAMP levels. The antioxidant and antiinflammatory action of forskolin is due to inhibition of macrophage activation with a subsequent reduction in thromboxane B2 and superoxide levels. These characteristics have made forskolin an effective medication for heart disease, hypertension, diabetes, and asthma. Here, we evaluated the effects of chronic forskolin administration on blood glucose and oxidative stress in 19 male Wistar rats with streptozotocin-induced diabetes compared to 8 healthy male Wistar rats. Rats were treated with forskolin, delivered daily for 8 weeks. Glucose was assessed by measuring fasting blood glucose in diabetic rats and with an oral glucose tolerance test (OGTT) in healthy rats. Oxidative stress was assessed by measuring 8 -hydroxydeoxyguanosine $(8-\mathrm{OHdG})$ in 24 -h urine samples. In diabetic rats, without forskolin, fasting blood glucose was significantly higher at the end than at the beginning of the experiment ( 8 weeks). In both healthy and diabetic rats, forskolin treatment lowered the fasting glucose at the end of the experiment but no effect was found on oral glucose tolerance. The 8-OHdG levels tended to be less elevated in forskolin-treated than in untreated group. Our results showed that chronic administration of forskolin decreased fasting blood glucose levels; however, the reductions of $8-\mathrm{OHdG}$ were not statistically significant.
\end{abstract}

Key words: Diabetes, 8-hydroxydeoxyguanosine, forskolin, glucose.

\section{Introduction}

Forskolin is a diterpene derived from plant Coleus forskohlii [1, 2]. Forskolin directly activates adenylate cyclase (AC), which increases intracellular cAMP levels in a variety of cells. The final effects of cAMP production are as diverse as the cells that respond to forskolin; the effects depend on the AC isoforms expressed in each kind of cell [3]. Previous research on pancreatic beta cells has shown that forskolin enhanced the glucose-mediated stimulus that induces beta cells to release insulin [4]. This effect was produced by the elevation of cAMP, which activates two main signaling pathways in beta cells. One pathway is mediated by protein kinase A (PKA) and the other is activated by factors of guanine nucleotide 
exchange that are regulated by cAMP [5].

Recent studies have shown that, in pancreatic islets cells, oxidative stress and glucotoxicity induced apoptosis [6]. Niaz \& Singh found that, in forskolin pre-treated erythrocytes, lipid peroxidation was significantly decreased [7]. Those authors concluded that the antioxidant effect of forskolin was comparable to the effects of vitamin $E$ and probucol. The increase in cAMP by forskolin attenuated cytotoxicity and apoptosis [6]. In vitro, forskolin protected against the intracellular effects of $\mathrm{H} 2 \mathrm{O} 2$ and increased the levels of the antioxidant, glutathione, by 1.6 to 2 fold [8].

Oxidative damage in vivo can be evaluated by measuring 8-hydroxydeoxyguanosine (8-OHdG), a nucleic acid that is modified in the presence of reactive oxygen species (ROS). The modified product (8-OHdG) can be measured in a $24-\mathrm{h}$ urine sample [9, 10].

Patients with diabetic nephropathy have displayed high levels of $8-\mathrm{OHdG}$, and $8-\mathrm{OHdG}$ was positively correlated with glycosylated hemoglobin $[11,12]$. High levels of $8-\mathrm{OHdG}$ were also correlated with indicators of diabetic complications, including the thickness of the intima of the carotid arteries and the calculated risk of coronary disease. Those data suggested that 8-OHdG was an early marker of microvascular and macrovascular type 2 diabetes complications; moreover, $8-\mathrm{OHdG}$ was a strong predictor of diabetic nephropathy progression $[11,13]$.

Studies have shown that drugs can modify oxidative stress, and these drugs were proven to be useful in diabetic mice [14]. It is also important to note that several studies have demonstrated the anti-inflammatory effects of forskolin. For example, when patients undergoing coronary bypass were administered a water soluble derivative of forskolin (colforsin; $0.5 \mu \mathrm{g} / \mathrm{kg} / \mathrm{min}$ ), they showed a reduced inflammatory response. Also, previous studies have shown that forskolin had an antagonistic effect on tumor necrosis factor alpha, and it reduced the levels of interleukins $1 \beta, 6$, and $8[9,15]$.

On the other hand, these antioxidant and anti-inflammatory action of forskolin has been used to treat the heart disease, hypertension, diabetes and asthma.

The aim of this study was to determine the effects of chronic administration of forskolin on glucose and oxidative stress in diabetic, male Wistar rats and on glucose tolerance in healthy, male Wistar rats.

\section{Materials and Methods}

\section{Experimental Animals}

Male Wistar rats, aged 10-14 weeks old, with a mean weight of $300 \mathrm{~g} \pm 50 \mathrm{~g}$, were obtained from
Harlan laboratories (Madison, WI, USA). The rats had free access to food and water and maintained at constant temperature of $22-24^{\circ} \mathrm{C}, 55 \%$ humidity, and a 12-h light/12-h dark cycle (lights on at 07:00h). Rats were managed in accordance with the Institute for Laboratory Animal Research Guide for the Care and Use of Laboratory Animals [16]. The Ethics Committee of the University Center for Biomedical Research, University of Colima, Colima, México, approved the protocol. After the experiments, the rats were anesthetized prior to euthanasia.

\section{Study Design}

A total of 27 rats were divided into four groups; 19 were experimentally induced to develop diabetes, and 8 were maintained in a healthy condition. Both diabetic and healthy rats received no forskolin (control), or $6 \mathrm{mg} / \mathrm{kg}$ per day of forskolin, administered orally for 8 weeks. Blood glucose levels were determined in each group before and after forskolin treatment. The diabetic rats were tested two weeks after confirming the presence of diabetes (three weeks after the induction) and after eight weeks of the designated treatment.

\section{Diabetes Induction}

Diabetes was induced with $60 \mathrm{mg} / \mathrm{kg}$ streptozotocin (Sigma ${ }^{\circledR}$, with citrate buffer at $\mathrm{pH} 4.5$ ) administered in a single dose intraperitoneally. The presence of diabetes was confirmed one week after induction. Blood glucose levels were determined, and induction was considered successful when blood glucose levels were $\geq 200 \mathrm{mg} / \mathrm{dl}$.

\section{Forskolin dose and administration}

Forskolin (10 mg capsules, Life Extension Quality Supplements and Vitamins, Inc., Ft Lauderdale Florida) was administered orally for 8 weeks by catheter (Industrial medical Plastica Silice Sa de CV, México). Considering the allometric scaling of rat metabolism, according to Duff [17], the administered doses $6 \mathrm{mg} / \mathrm{kg}$ per day was equivalent to the $1 \mathrm{mg} / \mathrm{kg}$ per day in humans doses. Forskolin was diluted in plain water to $60 \mathrm{mg} / 100$. We used the same forskolin doses and dilutions for diabetic and healthy rats.

\section{Glycemia Assessments}

Fasting blood glucose was measured in all rat groups at the beginning of the experiment and at the end of the experiment ( 8 weeks). Prior to each glucose measurement, rats underwent $8 \mathrm{~h}$ of overnight fasting [18]. All blood samples $(0.1 \mu \mathrm{l})$ were collected from the distal end of the tail and analyzed immediately with an Accu-check Active ${ }^{\circledR}$ glucometer and the corresponding Accu-check Active ${ }^{\circledR}$ test strips (Roche, Mannheim, Germany). 
The oral glucose tolerance test (OGTT) was administered to healthy rats at the beginning and at the end of the experiment. After $8 \mathrm{~h}$ overnight fast, glucose $(2 \mathrm{~g} / \mathrm{kg})$ was given orally, and blood glucose levels were measured, as described above, at 0, 30, 60, and $120 \mathrm{~min}$ after glucose loading.

\section{8-Hydroxydeoxyguanosine Assessments}

Oxidative stress was assessed by measuring 8-OHdG levels in 24-h urine samples in all rat groups at the beginning and end of the experiment (8 weeks). Urine samples were collected in the metabolic cages, frozen at $-70{ }^{\circ} \mathrm{C}$, and analyzed with an ELISA Kit (Trevigen, Gaithersburg, MD, USA).

\section{Statistical Analysis}

Averages, standard errors, and percentages were determined. Comparisons between average values were performed with the unpaired and the two tailed paired Student $\mathrm{t}$ test. In all tests, a confidence interval of $95 \%(95 \% \mathrm{CI})$ was used, and differences were considered statistically significant when $p$-values were < 0.05 .

\section{Results}

First, we examined the oral glucose tolerance curves for healthy rats treated with forskolin (Figure $1)$. The average blood glucose in the healthy rat group was $102.12 \pm 1.94 \mathrm{mg} / \mathrm{dl}, 101.25 \pm 3.56$ for control group and $103 \pm 2.08$ in forskolin group. The data showed that glucose levels at the end of the study are lower in forskolin group, with a significant difference according to the statistical tests applied $(p=0.03)$.

Next, we examined blood glucose levels of diabetic rats. At the beginning of the study, the mean blood glucose levels in diabetic rats were $278.7 \pm 18.6 \mathrm{mg} / \mathrm{dl}$ for the control (untreated) group; and $378.1 \mathrm{mg} / \mathrm{dl} \pm 40.10$ for rats that received forskolin. The within-group analyses showed a significant difference between initial and final glucose levels in the control group $(\mathrm{p}<0.001)$ (Figure 2).

We next determined the levels of urinary 8-OHdG. In the within-group analyses showed the same trend observed in blood glucose levels; the groups treated with forskolin exhibited less elevation in oxidative stress over time compared to the control group (Figure 3).

\section{Discussion}

In our experimental animals, forskolin predominantly decreased basal glucose in healthy rats and attenuated the severity of hyperglycemia in diabetic rats. The levels of 8-OHdG also tended to decrease in diabetic rats. These effects were observed after administering forskolin orally for 8 weeks.
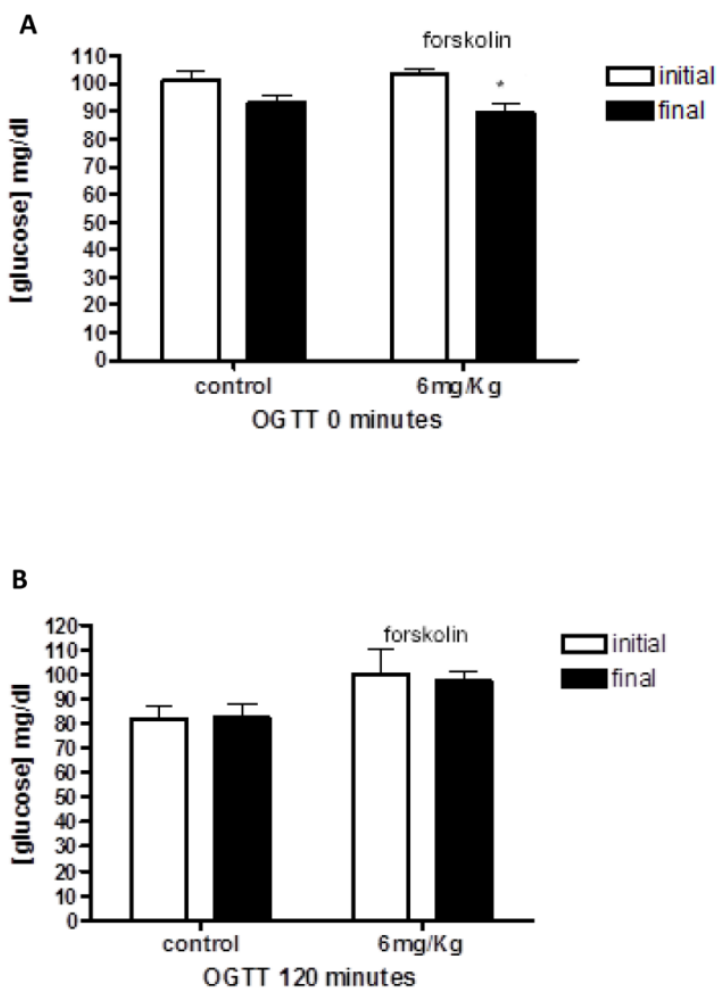

Figure I.The graphs illustrates baseline (A) and 120 (B) minutes oral glucose tolerance test on healthy rats at the beginning and end of treatment in the control group $(n=4)$ and forskolin $(n=4)$. Bars represent the means and line its standard error. $* \mathrm{p}<0.05$ (the initial measurement compared to the final measurement, statistic test paired $\mathrm{t}$-Student). OGTT = Oral Glucose Tolerance Test.

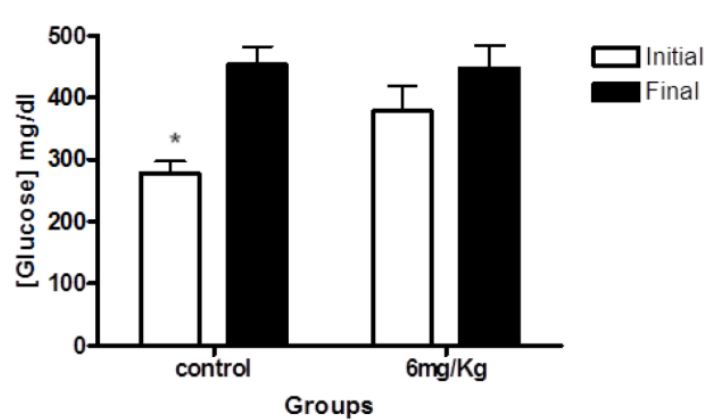

Figure 2. This graph illustrates glucose levels at baseline (white) and end of the study (black) in the control group $(n=10)$ and the experimental group (forskolin $n=9$ ). The bars represent the mean and line its standard error. $* p<0.05$ (the initial measurement compared to the final measurement, statistic test paired t-Student).

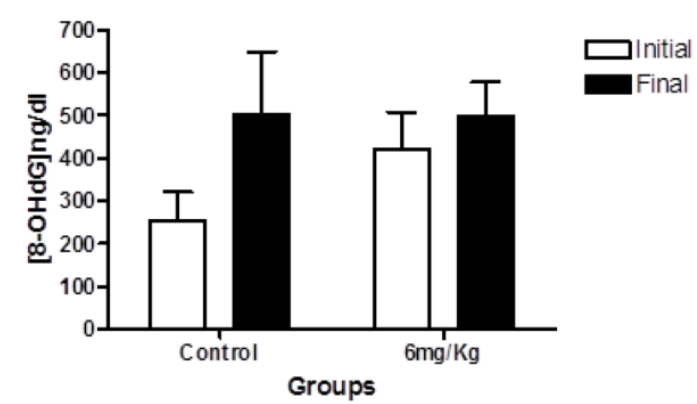

Figure 3. This graph indicates the levels of $8-O H d G$ in $24-$ h urine samples at baseline (white) and at endpoint (black) in the control group $(n=10)$ and experimental group (forskolin, $n=9$ ). Each bar represents the mean for each group and the lines represent standard error. No statistically significant difference was found between the baseline and the end $p>0.05$. 
It was previously shown that forskolin stimulated insulin release via elevation of intracellular cAMP in pancreatic beta cells $[4,19,20]$, that it conferred cytoprotection by decreasing glucotoxicity [6] and that it increased the expression of the insulin gene [21]. However, previously published results regarding the effects of forskolin on glucose levels are controversial. Ammon and Müller found that acute, intravenous administration of forskolin induced insulin release, increased cAMP in pancreatic beta cells, but elevated plasma glucose. However, when an intravenous glucose tolerance test was performed, glucose was not raised further than that observed in controls [4]. Ahmad et al. (1991) administered forskolin orally, as performed in this study. They delivered subchronic dosing and found that insulin and cAMP levels were elevated. They also reported an increase in glucose levels and an elevation in glucagon [19]. The effects of forskolin delivered in acute or subchronic doses were explained by: 1) elevation of glucagon, 2) increased gluconeogenesis, and 3) increased glycogenolysis [22].

Moreover, cAMP acutely increased insulin release by two mechanisms: 1) through PKA and a number of substrates, including the inositol phosphate- 3 receptor in the endoplasmic reticulum and the glucose transporter, GLUT2; and 2) the ATP-dependent $\mathrm{K}$ channel (KATP). A PKA-independent pathway also contributed to insulin release through an exchange protein that was directly activated by cAMP (EPAC). This pathway appeared to be activated when the PKA pathway was saturated, which is important for high performance in beta cells [20].

Here, we studied the chronic effects of forskolin. These effects may be due to a permanent elevation of cAMP in residual beta cells. Studies in other cells showed that, after systemic forskolin administration, the levels of cAMP were high compared to control cells (from untreated animals) [23]. It is also known that diabetic rats display a disorder in the AC-cAMP system that responds to glucose [24]. Thus, chronic administration of forskolin may induce a change in the balance of cAMP in response to glucose, which could enhance insulin release. This may be another mechanism for reducing glucotoxicity, in addition to the reduction of oxidative stress [6].

Chronic administration of GLP-1 agonists was found to induce insulin transcription and stabilize insulin levels [20]. Thus, in the present study, the effects on glucose levels, observed both in healthy and diabetic rats, may be explained by the effect of chronic forskolin on insulin transcription [21]. However, in healthy rats, the sensitivity to insulin did not change. This lack of response to insulin may be explained by the inhibitory effect of forskolin on glucose transporters, mainly Glut 4, which has a high affinity for glucose [25-30].

Our experimental findings showed no statistically significant difference in oxidative stress levels after forskolin treatment. However, we did observe a trend, similar to that observed in blood glucose levels, where the 8-OHdG values were lower in treated rats than in control rats at the end of the treatment period. These results were consistent with results described by Balkis Budin et al [31] and Ihara et al [32], who showed that oxidative DNA damage was negatively correlated with metabolic control in diabetic rats.

Several metabolic pathways are known to mediate the effects of chronic hyperglycemia on the production of ROS. One pathway is non-enzymatic glycosylation, which leads to the formation of advanced glycation end products, through intermediate products (Amadori); this pathway leads to the generation of ROS. Another pathway involves the electron transfer system, which becomes a major source of ROS. Therefore, we would expect these pathways to be activated in our chronically hyperglycemic rats [33].

On the other hand, in previous studies, forskolin was reported to have anti-inflammatory effects $[9,15]$. In this study, the results showed that forskolin affected oxidative stress. It is known that these two phenomena interact in several cardiovascular and metabolic pathologies, including diabetes [34, 35].

In summary, our results indicated that chronic administration of forskolin: 1) reduced serum levels of fasting glucose in healthy rats, 2) decreased the severity of fasting hyperglycemia in diabetic male Wistar rats, and 3) caused no statistically significant decrease in the 24-h urine levels of 8-OHdG. We note that the STZ method induces diabetes in rats on a faster timescale than the time it takes to develop diabetes in humans. Nevertheless, forskolin may be potentially useful in patients due to its mechanisms of action. It is possible that forskolin administration may have effects in humans that are qualitatively similar to those observed in the rat model of diabetes. However, further studies are needed to determine the proper dosages. In addition, previous studies have reported that the main adverse effect of forskolin was that it contributed to a reduction in blood pressure [2]. Susuki et al. also reported that forskolin was associated with tachycardia and cephalic pain [36]. Forskolin may also cause bleeding, due to its inhibitory effects on platelet adhesion [37, 38]. Future research in humans is needed to study potential adverse effects in patients with diabetes. 


\section{Abbreviations}

$\mathrm{AC}$, adenylate cyclase; cAMP, cyclic adenosine monophosphate; PKA, protein kinase A; 8- OHdG, 8,Hydroxydeoxyguanosine; STZ, streptozotocine; CTOG, oral glucose tolerance curve; ROS, reactive oxygen species; KATP, ATP-dependent $\mathrm{K}$ channel; EPAC, exchange protein activated by cAMP; GLP-1, glucagon-like peptide-1.

\section{Acknowledgements}

This work was partially funded by CONACyT-Mexico-MH 83313. Monica Rios- Silva had a PhD fellowship from CONACyT-Mexico. The authors wish to thank Jesus Dueñas for her technical assistance. This work was part of Monica Rios-Silva`s doctoral thesis at Medicine Faculty of the University of Colima (Mexico). Mónica Ríos-Silva, Benjamín Trujillo-Hernández, Miguel Huerta, Zorayda Urzúa, and Evelyn Mancilla designed and performed the experiments; Monica Ríos-Silva, Benjamín Trujillo-Hernández, Miguel Huerta, Zorayda Urzúa, Xóchitl Trujillo, and Enrique Sánchez-Pastor participated in the discussion and analysis of results; Mónica Ríos-Silva, Benjamín Trujillo-Hernández, Xóchitl Trujillo, Miguel Huerta, and Enrique Sánchez-Pastor wrote the manuscript. All authors are responsible for the content of the article. The authors declare no conflict of interest.

\section{Competing Interests}

The authors have declared that no competing interest exists.

\section{References}

1. Insel PA, Ostrom RS. Forskolin as a tool for examining adenylyl cyclase expression, regulation, and G protein signaling. Cell Mol Neurobiol. 2003; 23(3):305-314.

2. Bhat SV, Dohadwalla AN, Bajwa BS, Balbir S. , Nandkumar K. Dadkar NK, Dornauer H, De Souza NJ. The antihypertensive and positive inotropic diterpene forskolin: effects of structural modifications on its activity. J Med Chem. 1983; 26:486-492.

3. Pinto C, Papa D, Hübner M, Mou T, Lushington GH, Seifert R. Activation and inhibition of adenylyl cyclase isoforms by forskolin analogs. J Pharmacol Exp Ther. 2008; 325(1):27-36.

4. Ammon HP, Müller AB. Effect of forskolin on isle cyclic AMP, insulin secretion, blood glucose an intravenous tolerance in rats. Naunyn Schmiedebergs Arch Pharmacol. 1984; 326(4):364-367.

5. Holz GG. Epac: a new cAMP-binding protein in support of glucagonlike peptide-1 receptor-mediated signal transduction in the pancreatic betacell. Diabetes. 2004; 53:5-13.

6. Koh G, Suh KS, Chon S, Oh S, Woo JT, Kim SW, Kim JW, Kim YS. Elevated cAMP level attenuates 2-deoxy-d-ribose-induced oxidative damage in pancreatic beta-cells. Arch Biochem Biophys. 2005; 438(1):70-79.

7. Niaz MA, Singh RB. Modulation of free radical stress in human red blood cell membrane by forskolin and the prospects for treatment of cardiovascular disease and Diabetes. Cell Mol Biol (Noisy-le-grand). 1999; 45(8):1203-1207.

8. Kamata H, Tanaka C, Yagisawa H, Hirata H. Nerve growth factor and forskolin prevent $\mathrm{H} 2 \mathrm{O} 2$-induced apoptosis in PC12 cells by glutathione independent mechanism. Neurosci Lett. 1996; 212(3):179-182.

9. Loft S, Fischer-Nielsen A, Jeding IB, Vistisen K, Poulsen HE. 8-Hydroxydeoxyguanosine as a urinary biomarker of oxidative DNA damage. Toxicol Environ Health. 1993; 40(2-3):391-404.

10. Shigenaga MK, Gimeno CJ, Ames BN. Urinary 8-hydroxy-2'-deoxyguanosine as a biological marker of in vivo oxidative DNA damage. Proc Nall Acad Sci. 1989; 86:9697-9701.
11. Hinokio $Y$, Suzuki S, Hirai M, Suzuki C, Suzuki M, Toyota T. Urinary excretion of 8-oxo-7, 8-dihydro-2'-deoxyguanosine as a predictor of the development of diabetic nephropathy. Diabetologia. 2002; 45(6):877-882.

12. Xu GW, Yao QH, Weng QF, Su BL, Zhang X, Xiong JH. Study of urinary 8-hydroxydeoxyguanosine as a biomarker of oxidative DNA damage in diabetic nephropathy patients. J Pharm Biomed Anal. 2004; 36(1):101-104.

13. Nishikawa T, Sasahara T, Kirotoshi S, Sonoda K, Senokuchi T, Matsuo T, Kukidome D, Wake N, Matsumura T, Miyamura N, Sakakida M, Kishikawa $\mathrm{H}$, Araki E. Evaluation of urinary 8-Hydroxydeoxy-guanosine as a novel biomarker of macrovascular complications in type 2 diabetes. Diabetes Care, 2003; 26:1507-1512

14. Yoshida S, Hashimoto T, Kihara M, Imai N, Yasuzaki H, Nomura K, Kiuchi Y, Tamura K, Ishigami T, Hirawa N, Toya Y, Kitamura H, Umemura S. Urinary oxidative stress markers closely reflect the efficacy of candesartan treatment for diabetic nephropathy. Nephron Exp Nephrol. 2009; 111(1):20-30.

15. Hayashida N, Chihara S, Tayama E, Takaseya T, Enomoto N, Kawara T, Aoyagi S. Antiinflammatory effects of colforsin daropate hydrochloride, a novel water-soluble forskolin derivative. Ann Thorac Surg. 2001; 71(6):1931-1938.

16. [No authors listed] Institute for Laboratory Animal Research (ILAR): Guide for the Care and Use of Laboratory Animals. Washington: National Academy Press, 1996.

17. Duff G: Expert Scientific Group on Phase One Clinical Trials: Final Report. Norwich: TSO, 2006

18. Han BG, Hao CM, Tchkneva EE, Wang YY, Lee CA, Ebrahim B, Harris RC, Kern TS, Wasserman DH, Breyer MD, Qi Z. Markers of glycemic control in the mouse: comparisons of 6-h and overnight-fasted blood glucoses to $\mathrm{HbA1C}$. Am J Physiol Endocrinol Metab. 2008; 295:981-986.

19. Ahmad F, Khan MM, Rastogi AK, Kidwai JR. Insulin and glucagon releasing activity of coleonol (forskolin) and its effect on blood glucose level in normal and alloxan diabetic rats. Acta Diabetol Lat. 1991; 28(1):71-77.

20. Doyle ME, Egan JM. Mechanisms of action of GLP-1 in the pancreas. Pharmacol Ther. 2007; 113(3):546-593.

21. Goodison S, Kenna S, Ashcroft SJ. Control of insulin gene expression by glucose. Biochem J. 1992; 285(2):563-568.

22. Exton JH, Robison GA, Sutherland EW, Park CR. Studies on the role of adenosine $3^{\prime}, 5^{\prime}$-monophosphate in the hepatic actions of glucagon and catecholamines. J Biol Chem. 1971; 246(20):6166-6177.

23. Nomura Y, Arima T, Swgawa T. Influences of pertussis toxin, guanine nucleotides and forskolin on adenylate cyclase in striatal membranes of infant, adult and senescent rats. Int J Dev Neurosci. 1987; 5(3):271-279.

24. Dachicourt N, Serradas P, Giroix MH, Gangnerau MN, Portha B. Decreased glucose-induced cAMP and insulin release in islets of diabetic rats: reversal by IBMX, glucagon, GIP. Am J Physiol. 1996; 271(4 Pt 1):725-732.

25. Joost HG, Steinfelder HJ. Forskolin inhibits insulin-stimulated glucose transport in rat adipose cells by a direct interaction with the glucose transporter. Mol Pharmacol. 1987; 31(3):279-283.

26. Shanahan MF, Edwards BM, Ruoho AE. Interactions of insulin, catecholamines and adenosine in the regulation of glucose transport in isolated rat cardiac myocytes. Biochim Biophys Acta. 1986; 887(1):121-129.

27. Sergeant S, Kim HD. Inhibition of 3-O-methylglucose transport in human erythrocytes by forskolin. J Biol Chem. 1985; 260(27):14677-14682.

28. Kim HD, Sergeant S, Shukla SD. Glucose transport in human platelets and its inhibition by forskolin. J Pharmacol Exp Ther. 1986; 236(3):585-589.

29. Hellwig B, Joost HG. Differentiation of erythrocyte-(GLUT1), liver-(GLUT2), and adipocyte-type (GLUT4) glucose transporters by binding of the inhibitory ligands cytochalasin $\mathrm{B}$, forskolin, dipyridamole, and isobutylmethylxanthine. Mol Pharmacol. 1991; 40(3):383-389.

30. Kashiwagi A, Huecksteadt TP, Foley JE. The regulation of glucose transport by cAMP stimulators via three different mechanisms in rat and human adipocytes. J Biol Chem, 1983; 258(22):13685-13692.

31. Balkis Budin S, Othman F, Louis SR, Abu Bakar M, Radzi M, Osman K, Das S, Mohamed J. Effect of alpha lipoic acid on oxidative stress and vascular wall of diabetic rats. Rom J Morphol Embryol. 2009; 50(1):23-30.

32. Ihara $Y$, Yamada $Y$, Toyokuni S, Miyawaki K, Ban N, Adachi T, Kuroe A, Iwakura T, Kubota A, Hiai H, Seino Y. Antioxidant alpha-tocopherol ameliorates glycemic control of GK rats, a model of type 2 diabetes. FEBS Lett. 2000; 473(1):24-26

33. Kawahito S, Kitahata H, Oshita S. Problems associated with glucose toxicity: role of hyperglycemia-induced oxidative stress. World J Gastroenterol. 2009; 15(33):4137-4142.

34. Wadley AJ, Veldhuijzen van Zanten JJ, Aldred S. The interactions of oxidative stress and inflammation with vascular dysfunction in ageing: the vascular health triad. Age (Dordr). 2013; 35(3):705-718.

35. Guzik TJ, Korbut R, Adamek-Guzik T. Nitric oxide and superoxide in inflammation and immune regulation. J Physiol Pharmacol. 2003; 54(4):469-487.

36. Suzuki S, Ito O, Sayama T, Goto K. Intra-arterial colforsin daropate for the treatment of cerebral vasospasm after aneurysmal subarachnoid hemorrhage. Neuroradiology. 2010; 52(9):837-845.

37. Putnam WC, Swenson SM, Reif GA, Wallace DP, Helmkamp GM Jr, Grantham JJ. Identification of a forskolin-like molecule in human renal cysts. J Am Soc Nephrol. 2007; 18(3):934-943.

38. Christenson JT, Thulesius O, Nazzal MM. The effect of forskolin on blood flow, platelet metabolism, aggregation and ATP release. Vasa, 1995; 24(1):56-61. 